\title{
Increasing the Selectivity of Pt-Gate SiC Field Effect Gas Sensors by Dynamic Temperature Modulation
}

Christian Bur, Peter Reimann, Mike Andersson, Andreas Schuetze and Anita Lloyd Spetz

\section{Linköping University Post Print}

N.B.: When citing this work, cite the original article.

C2012 IEEE. Personal use of this material is permitted. However, permission to reprint/republish this material for advertising or promotional purposes or for creating new collective works for resale or redistribution to servers or lists, or to reuse any copyrighted component of this work in other works must be obtained from the IEEE.

Christian Bur, Peter Reimann, Mike Andersson, Andreas Schuetze and Anita Lloyd Spetz, Increasing the Selectivity of Pt-Gate SiC Field Effect Gas Sensors by Dynamic Temperature Modulation, 2012, IEEE Sensors Journal, (12), 6.

http://dx.doi.org/10.1109/JSEN.2011.2179645

Postprint available at: Linköping University Electronic Press

http://urn.kb.se/resolve?urn=urn:nbn:se:liu:diva-77724 


\title{
Increasing the Selectivity of Pt-gate $\mathrm{SiC}$ Field Effect Gas Sensors by Dynamic Temperature Modulation
}

\author{
Christian Bur, Peter Reimann, Mike Andersson, Andreas Schütze, and Anita Lloyd Spetz
}

\begin{abstract}
Based on a diode coupled silicon carbide field effect transistor with platinum as catalytic gate material, the influence of dynamic temperature modulation on the selectivity of GasFETs has been investigated. This operating mode, studied intensively for semiconductor gas sensors, has only recently been applied to field effect transistors. A suitable temperature cycle (T-cycle) for detection of typical exhaust gases $\left(\mathrm{CO}, \mathrm{NO}, \mathrm{C}_{3} \mathrm{H}_{6}, \mathrm{H}_{2}, \mathrm{NH}_{3}\right.$ ) was developed and combined with appropriate signal processing. The sensor data was evaluated using multivariate statistics, e.g. linear discriminant analysis (LDA). Measurements have proven that typical exhaust gases can be discriminated in backgrounds with $0 \%, 10 \%$ and $20 \%$ oxygen. Furthermore, we are able to quantify the mentioned gases and to determine unknown concentrations based on training data. Very low levels of relative humidity (r.h.) below a few percent influence the sensor response considerably but for higher levels the cross interference of humidity is negligible. In addition, experiments regarding stability and reproducibility were performed.
\end{abstract}

Index Terms-MISiC FET, NOx, selectivity, temperature modulation, virtual multi sensor

\section{INTRODUCTION}

$\mathrm{A}^{1}$ LONG with the debate about climate change, it has become more and more important to control the emissions of environmentally hazardous and greenhouse gases, e.g. from cars or other combustion processes. Thus, small, fast and especially cheap sensors have become of interest but the demands on the sensor/sensor system are fairly high. For instance, in the automotive industry for a continuous

Manuscript received October 1, 2011. This work was supported in part by the Swedish Research Council, the Swedish Agency for Innovation Systems (VINNOVA), and Swedish Industry through the VINN Excellence Centre FunMat.

Christian Bur is enrolled in a joint Ph.D. program with Saarland University, D-66123 Saarbrücken, Germany and Linköping University, S58183 Linköping, Sweden (corresponding author: phone: 0049 681/3023904; fax: 0049 681/302-4665; e-mail: c.bur@LMT.uni-saarland.de).

Peter Reimann was with the Laboratory for Measurement Technology, Saarland University, D-66123 Saarbrücken, Germany. He finished his Ph.D. in spring 2011.

Andreas Schütze is with the Laboratory for Measurement Technology, Saarland University, D-66123 Saarbrücken, Germany.

Mike Andersson is with the Div. of Applied Physics, Linköping University, S-58183 Linköping, Sweden.

Anita Lloyd Spetz is 50\% with the Div. of Applied Physics, Linköping University, Sweden and 50\% with the Microelectronics and Material Physics Laboratories, Oulu University, Finland. monitoring of the emissions from cars a sensor response in the order of milliseconds [1] is necessary; in addition, the sensor has to withstand harsh environments with high temperatures and corrosive atmospheres.

The semiconductor silicon carbide $(\mathrm{SiC})$ is a suitable material for realizing sensors for these kinds of applications. A wide band gap of $3.2 \mathrm{eV}$ in the case of $4 \mathrm{H}$ silicon carbide allows operating temperatures up to $700^{\circ} \mathrm{C}$ for short periods of time without losing its semi-conductive behavior. In addition, the high chemical inertness of $\mathrm{SiC}$ allows application in harsh environments. Various different types of $\mathrm{SiC}$ based sensors have been suggested in the last years like MOS capacitors [2] and Schottky diodes [3]. The advantages of both types can be combined in a field effect transistor (FET). Using a catalytic gate material like palladium, platinum or iridium the device can be made gas sensitive [4]. Metal insulator silicon carbide field effect transistors (MISiC FET), especially, seem to be suitable candidates for emission control and on board diagnostics (OBD) [5]. Since the introduction of selective catalytic reduction (SCR) of nitrogen oxides $\left(\mathrm{NO}_{\mathrm{x}}\right)$ by ammonia $\left(\mathrm{NH}_{3}\right)$ in diesel engines [6], the detection of either ammonia $\left(\mathrm{NH}_{3}\right)$ [7] or nitrogen oxides $\left(\mathrm{NO}_{\mathrm{x}}\right)$ [8], [9] have received increasing interest. Another possible automotive application of a MISiC FET is as a lambda-sensitive device for enhancing the efficiency of the catalytic converter [10]. For these applications, it was reported that the sensor response time of a MISiC FET is below $100 \mathrm{~ms}$ for ammonia exposure (at $300^{\circ} \mathrm{C}$ ) and below $20 \mathrm{~ms}$ for hydrogen $\left(\right.$ at $550^{\circ} \mathrm{C}$ ) which exceeds requirements considerably [1]. The MISiC FET is also commercialized as a sensor system for control of domestic boilers [11], [12].

However, measuring and discriminating exhaust gases are still challenging tasks [13] due to similar reactions of the sensor to various gases. In the last years, much effort has been spent on increasing the selectivity of the sensors by finding new materials, material compositions or sensor setups.

The lack of selectivity and poor long time stability are wellknown drawbacks of chemical sensors. In many cases sensor arrays offer better selectivity but lead to increasing costs as well as further reduced stability. For semiconductor (SC) gas sensors it was shown that temperature cycling is a powerful approach for increasing both selectivity and stability of the 
sensor [14]. The most promising technique is a varying heater voltage covering a broad temperature range [15]. In reviews by Lee et al. [16] and Sears et al. [17], [18] excellent summaries of some advantages of temperature cycled operation (TCO) are given. A varying sensor temperature leads to a unique signature for each gas due to different rates of reaction and sensor response to various gases. Furthermore, a low operating temperature, while achieving high sensitivity for some gases like $\mathrm{CO}$, often causes an incomplete reaction on the sensor surface and thus, an accumulation of incompletely oxidized components which requires periodic heating up to higher temperatures to clean the surface. Finally, the sensitivity can be improved because for each gas there is a specific temperature corresponding to a maximum of the sensor response.

The TCO mode makes a more comprehensive signal processing necessary. The obtained multi-dimensional data are usually evaluated by pattern recognition techniques, e.g. by linear discriminant analysis (LDA) [19]. A system based on a single gas sensor (or at most a few) run by dynamic temperature modulation and pattern recognition afterwards is sometimes called a virtual multisensor [20] because it combines advantages from both a single sensor (stability) and a sensor array (selectivity) [14].

In this paper, dynamic temperature modulation is applied to MISiC FET gas sensors in order to investigate the suitability of the virtual multisensor approach.

\section{EXPERIMENTAL}

\section{A. Sensor Device}

The device used in this work is based on the MOSFET technology with $\mathrm{SiC}$ as substrate material. A schematic

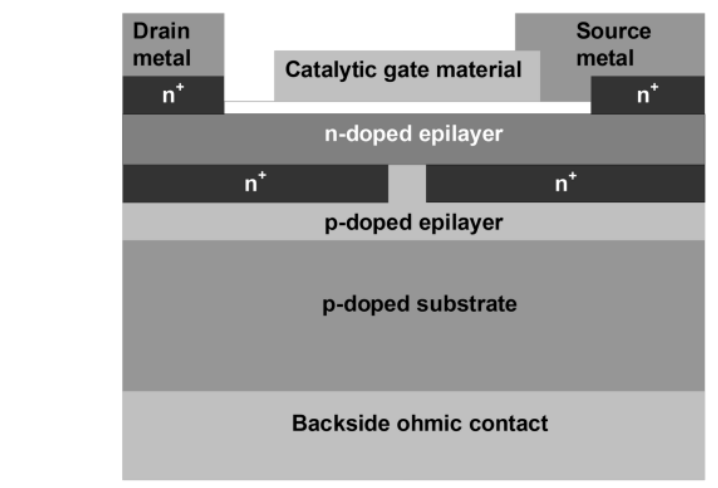

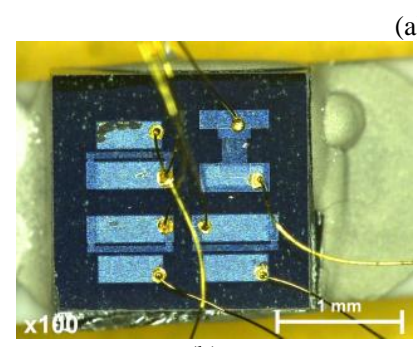

(b)

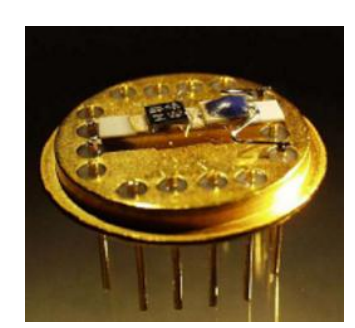

(c)
Fig. 1 (a) Device cross section of the used depletion type MISiC FET. (b) Top view of the sensor chip. (c) View of the device mounted with ceramic heater and temperature sensor on a TO8 header. drawing is shown in Fig. 1a. As can be seen in the figure the gate and source contacts of the device are connected in order to obtain a simple two-terminal device. The transistor is operated at a constant current of $100 \mu \mathrm{A}$ and the resulting drain/gate to source voltage is defined as the sensor signal. This voltage drop is influenced in the presence of various reducing and oxidizing gases. The response mechanism for hydrogen containing gases is mainly based on formation of polar hydroxide groups on the insulator surface at the metal insulator interface [4], [21]. Non-hydrogen containing dipole gases like $\mathrm{CO}$ and NO may also directly form dipoles or charged species on uncovered patches of the insulator [22], [23].

The sensors were processed on p-type $\mathrm{SiC}$ wafers and divided into single chips holding three sensors (diode-coupled transistors) and one test structure (not used in this project). The chip size is 2.1 by $1.9 \mathrm{~mm}^{2}$. The device was designed and fabricated at Linköping University together with ACREO AB, Kista, Sweden [24]. The catalytic gate material is a porous film of platinum which was deposited by sputter-deposition in an argon atmosphere.

The sensor chip is glued together with a Pt-100 temperature sensor on a ceramic heater (Heraeus [25]) and mounted on a 16-pin holder as shown in Fig. 1b, c.

\section{B. Temperature Cycle}

Our selected temperature cycle (T-cycle) covers a temperature range from $100^{\circ} \mathrm{C}$ to $330^{\circ} \mathrm{C}$ which approximately matches the points of maximal sensitivity of the used gases. In applications where the ambient temperature is significantly higher, e.g. directly in the exhaust stream, the temperature range of the T-cycle would have to be adjusted accordingly. The T-cycle presented here is suitable under laboratory conditions and is only used to prove the general suitability of TCO for GasFET sensors.

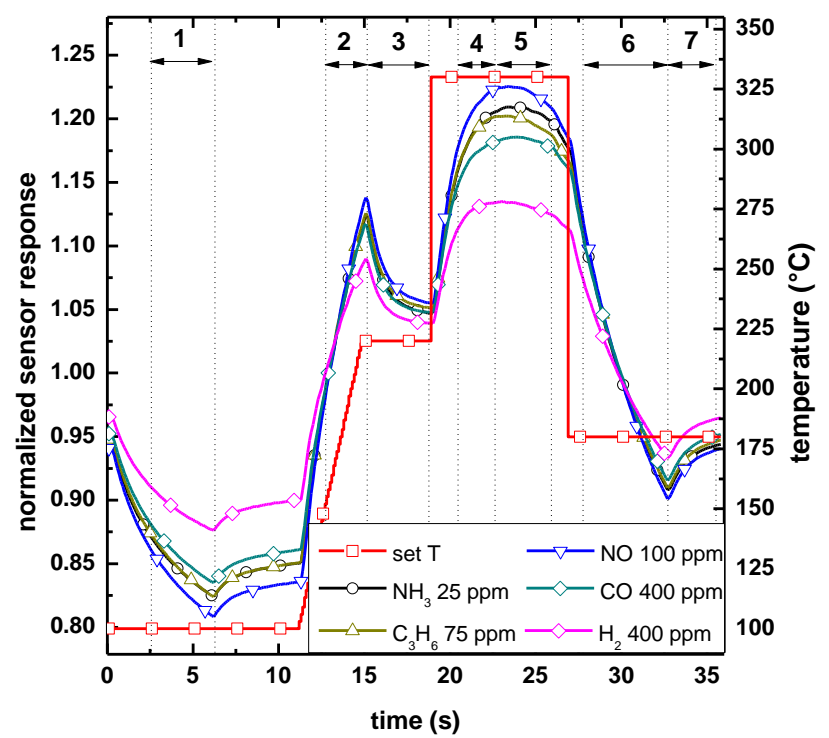

Fig. 2 Applied temperature cycle (squares) and the resulting normalized dynamic sensor response for $\mathrm{NH}_{3}, \mathrm{C}_{3} \mathrm{H}_{6}, \mathrm{NO}, \mathrm{CO}$ and $\mathrm{H}_{2}$. Seven intervals are marked for feature extraction. 


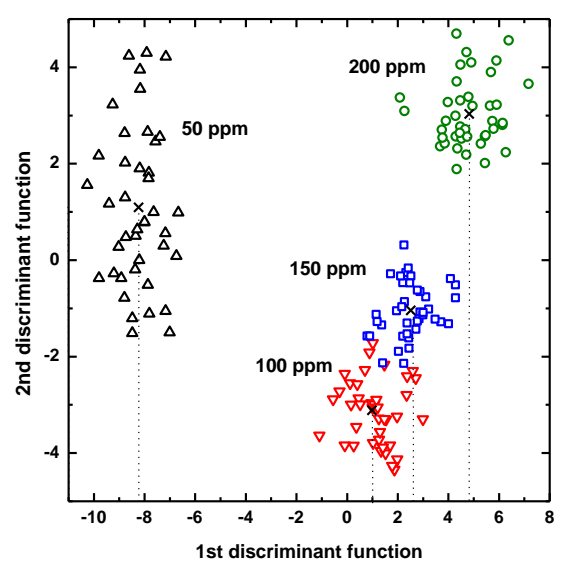

(a)

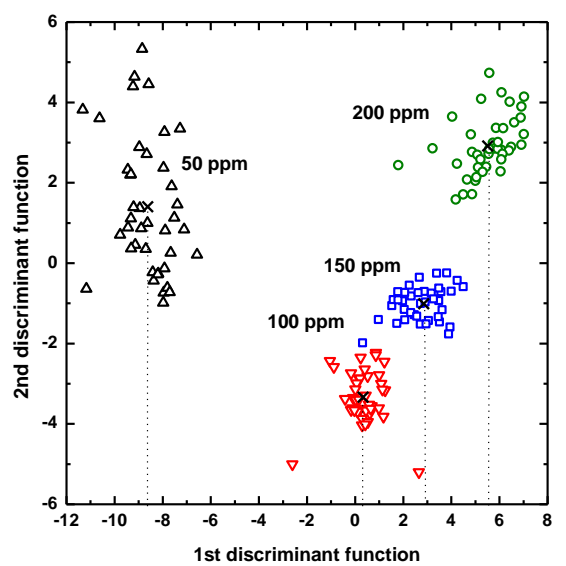

(b)

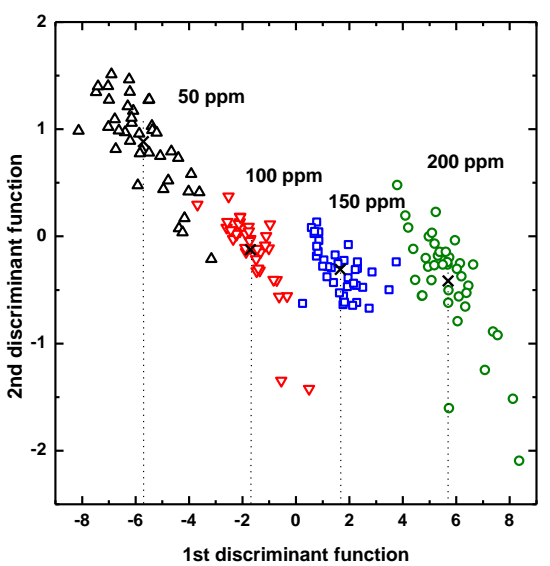

(c)

Fig. 3 LDA plots showing the influence of the signal processing on the quantification of $\mathrm{NH}_{3}$ in an atmosphere with $10 \%$ oxygen. (a) Only the slope and mean value in the seven intervals were used for discrimination. (b) The sensor raw data have been smoothed by SG filter before feature extraction. (c) Additional features like the standard deviation and the Euclidean norm of the data point vector were used for discrimination after smoothing with SG filter.

In order to allow dynamic temperature modulation the operating temperature is set using a PID controller implemented in a microcontroller using the Pt-100 signal as process variable.

The duration of the cycle consisting of a ramp and some steps, is $35.6 \mathrm{~s}$ which is due to the thermal time constant of the ceramic heater which is in the order of $4-5 \mathrm{~s}$. Fig. 2 shows the temperature set points (squares) and the resulting, normalized sensor responses to various gas exposures. The overshoot in the dynamic sensor response when reaching the first temperature plateau (i.e. $220^{\circ} \mathrm{C}$ ), is probably caused by the temperature control loop which uses the additional Pt-100 temperature sensors as input signal. Thus, the actual temperature on the sensor surface may differ from the set value.

\section{Used Gases}

The gases used in this project are typical for automotive or power plant exhausts. Since introducing SCR systems the detection of $\mathrm{NH}_{3}$ has become of interest and is therefore investigated as well. For almost all the measurements five gases $\left(\mathrm{H}_{2}, \mathrm{CO}, \mathrm{NO}, \mathrm{C}_{3} \mathrm{H}_{6}\right.$ and $\left.\mathrm{NH}_{3}\right)$ with four concentrations per gas are applied to the sensor. The flow over the sensor was kept constant at $100 \mathrm{ml} / \mathrm{min}$ and the exposure duration of each gas concentration was $1800 \mathrm{~s}$. All measurements presented in this paper were done in nitrogen atmosphere, sometimes with admixture of oxygen and additional water vapor.

\section{Signal PROCESSING}

The multidimensional data coming from the sensor was evaluated by using pattern recognition tools, e.g. linear discriminant analysis (LDA). As input to the LDA model gas significant features describing the shape [26] of the sensor response have been extracted in seven intervals of the T-cycle (marked in Fig. 2). In order to make the features more robust towards noise, the sensor response was smoothed using a Savitzky-Golay Convolution filter [27] in a first step. After that, the smoothed sensor data have been normalized by dividing each data point by the cycle mean value to reduce baseline drift and therefore to make the sensor more stable [20]. The normalized dynamic sensor response (one selected cycle per gas) is shown in Fig. 2.

In addition to the established standard features (like slope and mean value) [20], the Euclidean norm and the standard deviation were used as gas significant features [28].

The influence of the advanced signal processing is shown in Fig. 3 by taking the example of discrimination of $\mathrm{NH}_{3}$ in an atmosphere with $10 \%$ oxygen. In (a) the sensor data were normalized (without smoothing) and only the standard features are used for classification. Quantification is possible even though the scatter within the groups is relatively high. If the data are additionally smoothed, the quality of the discrimination can be enhanced (b). Using the Euclidean norm and the standard deviation in addition to the standard features, a linear dependency of the value of the first discriminant function on the concentration is obtained.

\section{RESULTS AND DISCUSSION}

In this section, results showing the influence of temperature modulation on the sensor response are given. We start by presenting results of quantitative analysis of the mentioned test gases and continue by investigating the influence of oxygen and humidity on the discrimination. A special focus is set on ammonia detection since its importance for SCR systems. We conclude with some remarks on stability and reproducibility of our suggested approach.

\section{A. Quantitative Analysis}

In a first step, quantitative analysis was carried out. This means focusing the LDA on different concentrations of one particular test gas in order to discriminate different concentrations. Fig. 4a shows the resulting two dimensional LDA plot for different concentrations of CO. The groups representing different concentrations are well separated making discrimination possible. It can easily be observed that there is a good correlation between the $\mathrm{CO}$ concentration and 


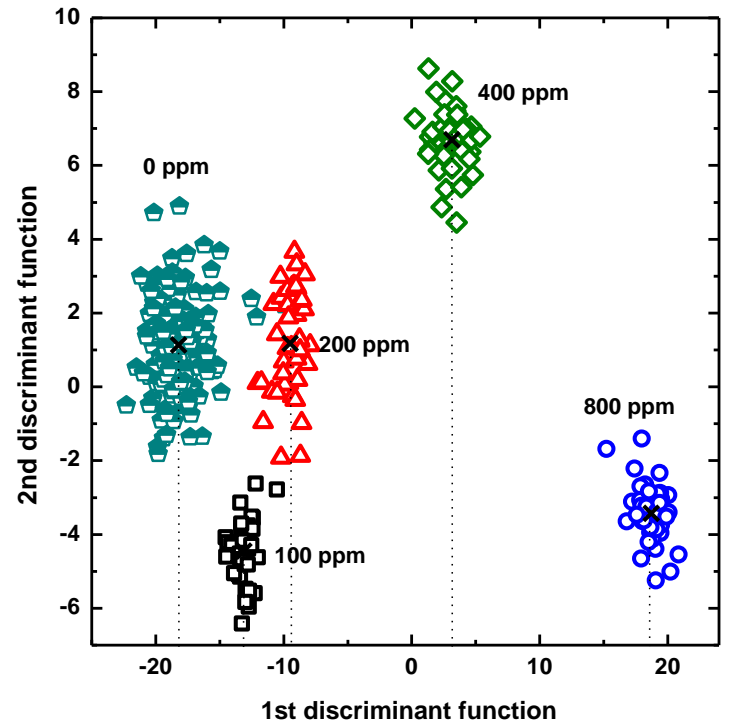

(a)

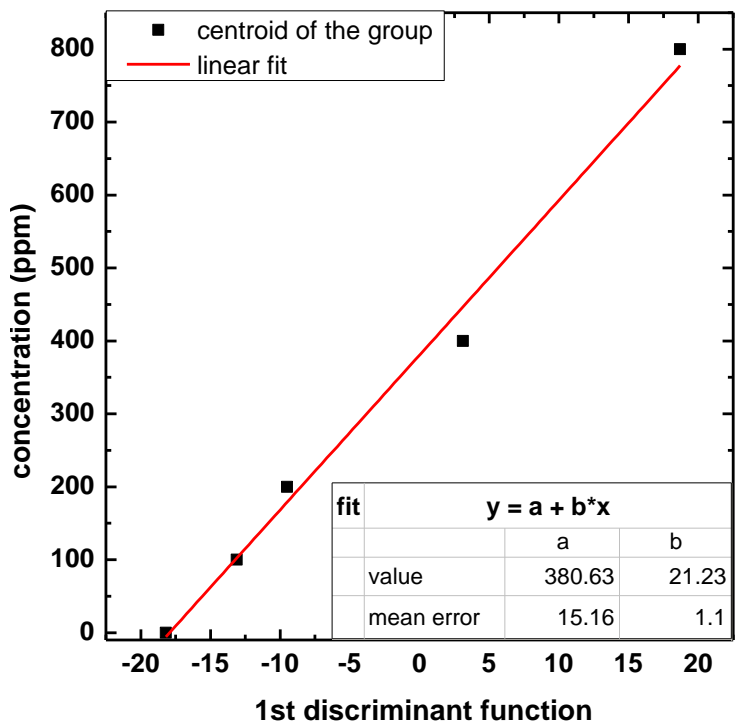

(b)

Fig. 4 Correlation between gas concentration and first discriminant function (DF1), here shown for CO in $\mathrm{N}_{2}$. (a) LDA plot of different $\mathrm{CO}$ concentrations, (b) concentration as function of DF1 with linear fit.

the first discriminant function (DF1). In order to prove the mentioned correlation between the concentration and the first $\mathrm{DF}$, the centroid of each class is fitted by a first order polynomial (Fig. 4b) obtaining an almost linear behavior. For various applications this result is very interesting because an unknown $\mathrm{CO}$ concentration can thus easily be predicted based only on the value of the first DF.

The calculated LDA coefficients were in a second step applied to data obtained in pure nitrogen (i.e. $0 \mathrm{ppm} \mathrm{CO}$ ). The resulting group is projected in Fig. $4 \mathrm{a}$ as well (half-solid symbols). Even this new class fits into the concentration proportionality of the first four groups as shown in Fig. $4 \mathrm{~b}$.

\section{B. Influence of Background Oxygen}

In addition to quantitative analysis, i.e. determination of the concentration of one particular gas, separation of the various gases under different conditions was studied. Fig. 5a and b

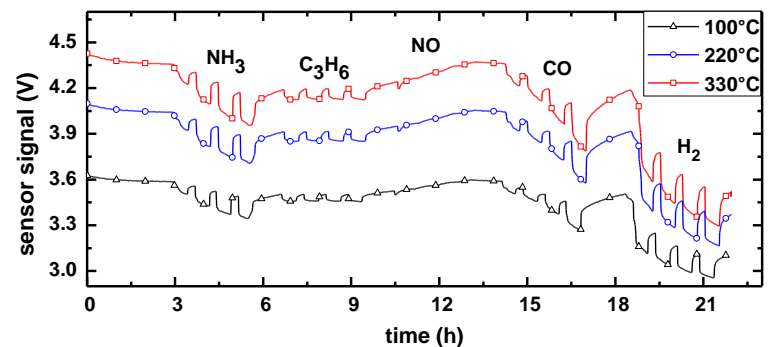

(a)

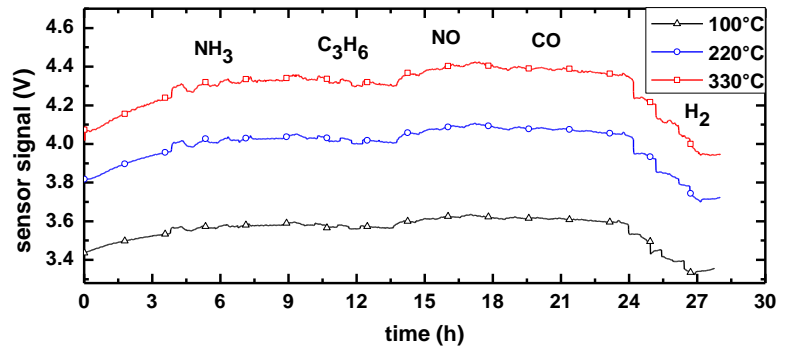

(b)

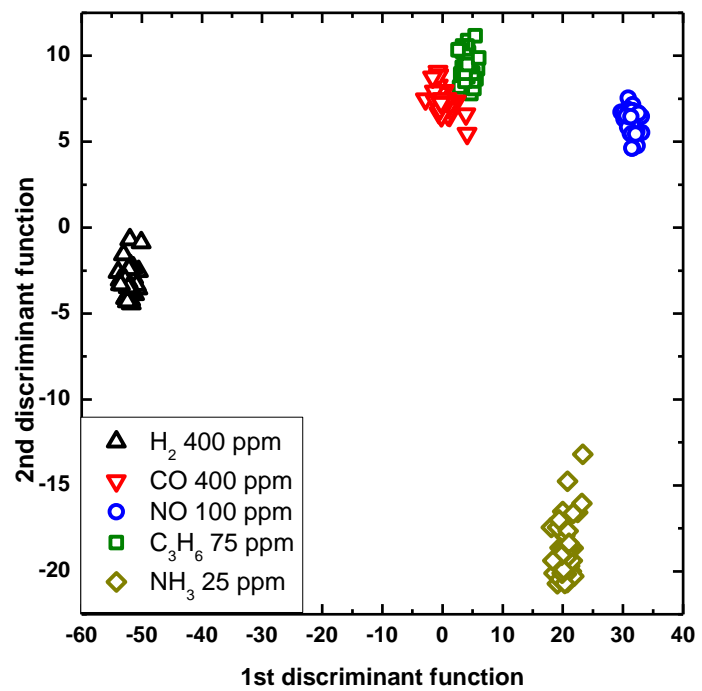

(c)

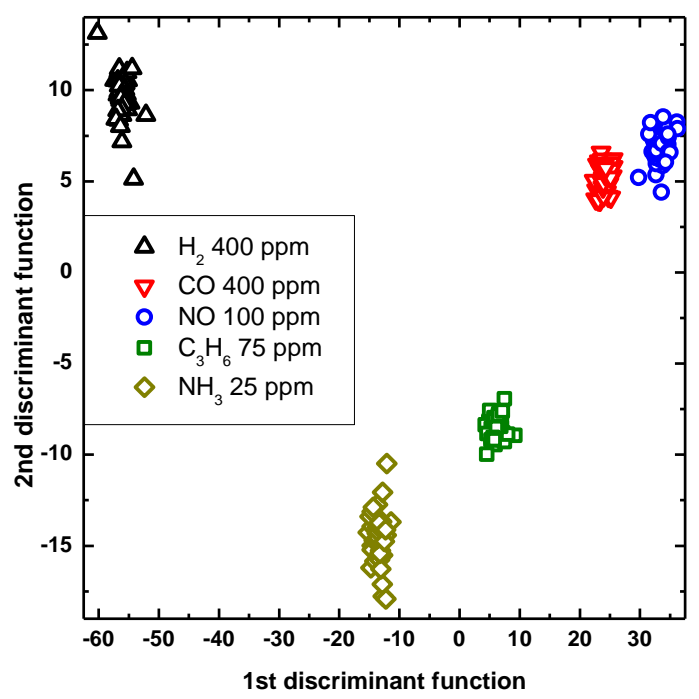

(d)

Fig. 5 Quasi static sensor reaction (a) in pure nitrogen, (b) with $20 \%$ oxygen in the background and corresponding LDA plots with one concentration per gas (c) in pure nitrogen, (d) in $20 \%$ oxygen. Measurements were performed in dry atmosphere. 
show quasi static sensor reactions of the used sensor towards the mentioned test gases. The expression "quasi static" means that from each T-cycle of the entire measurement only one sampling point (corresponding to a specific temperature) is evaluated. In these figures, temperature levels of $100^{\circ} \mathrm{C}$, $220^{\circ} \mathrm{C}$ and $330^{\circ} \mathrm{C}$ are shown (compare T-cycle shown in Fig. 2). It can be observed that without oxygen in the background (Fig. 5a) the sensor signal decreases for all investigated gases except for NO. Compared to the sensor response in oxygen atmosphere (Fig. 5b) the response is much stronger in pure nitrogen. It is interesting to note that the sensor reaction to $\mathrm{NH}_{3}$ and $\mathrm{C}_{3} \mathrm{H}_{6}$ changes its sign when oxygen is present. This can be explained by the sensing mechanism now being dominated by the reaction of the gas with adsorbed oxygen and spill-over effects [4], [21].

By looking at the results coming from the linear discriminant analysis one can say separation of these typical exhaust gases works pretty well (Fig. 5c, d) due to the low scatter within the groups and the clearance between the groups. Especially the temperature reaction to hydrogen and ammonia is quite different from the other gases $\left(\mathrm{CO}, \mathrm{NO}\right.$ and $\left.\mathrm{C}_{3} \mathrm{H}_{6}\right)$. Comparing the results in nitrogen to the measurement with $20 \%$ oxygen in the background, the distribution of the classes is not affected greatly. Only the group for $\mathrm{C}_{3} \mathrm{H}_{6}$ exposure is shifted in the direction of the $\mathrm{NH}_{3}$ group. All in all, the pattern contained in measurement data and described by the extracted features does not alter significantly with varying oxygen although the absolute values of the sensor response are fairly different.

\section{Influence of Water Vapor}

Besides the dependence of the sensor signal from the background oxygen concentration the amount of water vapor contained in the gas mixture affects the sensor behavior. Especially for metal oxide sensors the level of humidity is not

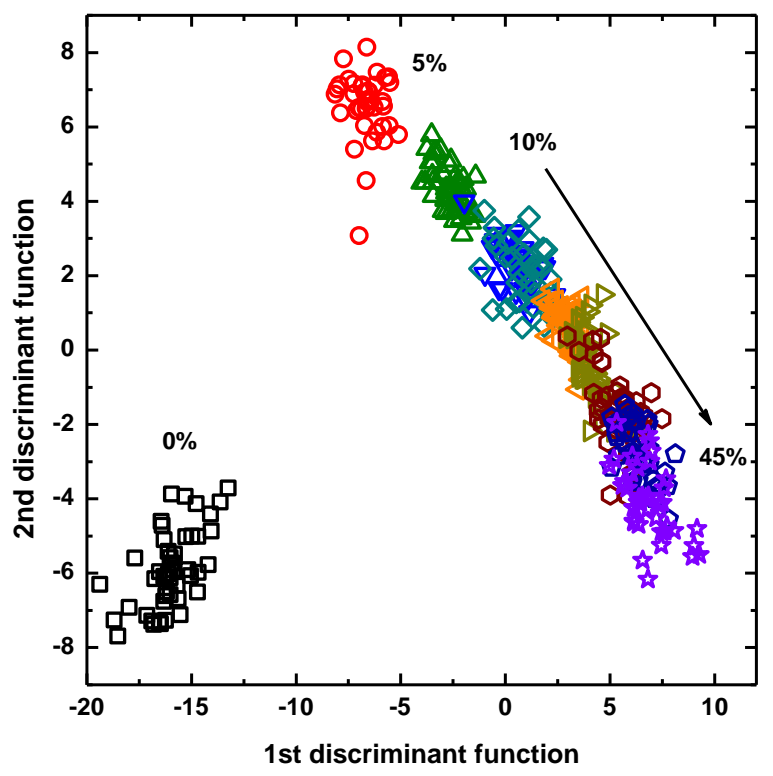

Fig. 6 LDA plot showing the influence of rel. humidity on the discrimination of $750 \mathrm{ppm} \mathrm{H}_{2}$ in synthetic air. negligible [29]. It is known that the influence of humidity on the sensing properties of field effect gas sensors is not as strong as for metal oxide gas sensors. It was reported that for MISiC-FETs the influence of water vapor is constant for humidity levels above 2\% [4], [7] while there is strong humidity dependence for MOX sensors over practically the whole range [29], [30]. However, the influence was studied again due to the fact that the FET gas sensor is now run in TCO. Thus, the sensor was exposed to $750 \mathrm{ppm}$ of $\mathrm{H}_{2}$ in synthetic air while the level of relative humidity (r.h.) is increased from $0 \%$ up to $45 \%$ in 5\% steps. The resulting LDA plot is given in Fig. 6. Obviously the main variation is between dry hydrogen (i.e. $0 \%$ rel. hum.) and humidified hydrogen. The strong overlap of the groups corresponding to higher levels of humidity (> 20\%) indicates that the influence of a changing amount of water vapor is relatively weak and does not influence the behavior much. Most applications, e.g. combustion processes, usually have a higher level of humidity. Our used GasFET combined with signal processing based on pattern recognition is almost insensitive to changes in the amount of water vapor contained in the gas mixture. For applications in dry atmospheres or at very low levels of humidity, i.e. in the critical range for MISiC FET (below 2\%), a hierarchical strategy as described in [9] could be used to reduce the influence of the background (e.g. humidity level or oxygen conc.) so that there is no need for additional sensors.

\section{Ammonia Measurements}

According to the relevance of measuring ammonia in SCR systems, quantitative analysis of $\mathrm{NH}_{3}$ was investigated more intensively. Thus, the concentration of $\mathrm{NH}_{3}$ was increased in $25 \mathrm{ppm}$ steps from $50 \mathrm{ppm}$ to $225 \mathrm{ppm}$. In Fig. 7a the LDA was only trained with three different $\mathrm{NH}_{3}$ concentrations (50, 125, 200 ppm, marked by solid symbols).

In a second step it was checked if a prediction of unknown concentrations is possible based on this three point calibration. The obtained coefficients from the LDA were used for projecting the unknown concentrations of ammonia $(75,100$, $150,175,225 \mathrm{ppm})$. Evidently, the new groups fit perfectly into the training set. This phenomenon is highlighted in Fig. 7b where the value of the first DF is plotted vs. concentration. The centroids of the training groups (marked by stars) are then fitted by a second order polynomial showing an almost linear behavior, similar to Fig. 3c or Fig. 4a. The centroids of the projected groups (evaluation data) are also plotted in Fig. $7 \mathrm{~b}$ (marked by solid squares). Due to the small distance between the projected groups and the fitted curve, one can conclude that it is sufficient to train the system with only a few concentrations while determination of other concentrations is still achievable. Hence, a prediction of unknown concentrations is possible with this approach.

\section{E. Verification of the T-cycle Approach}

Using pattern recognition to evaluate measurement data sometimes leads to wrong results (although they look quite well) due to an over-fitting of the extracted features, for 


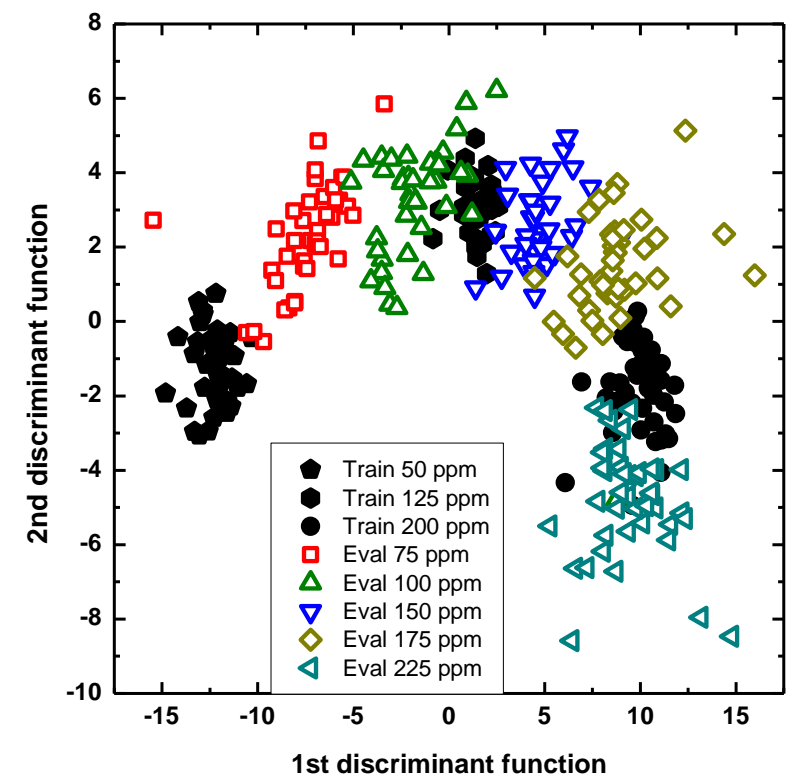

(a)

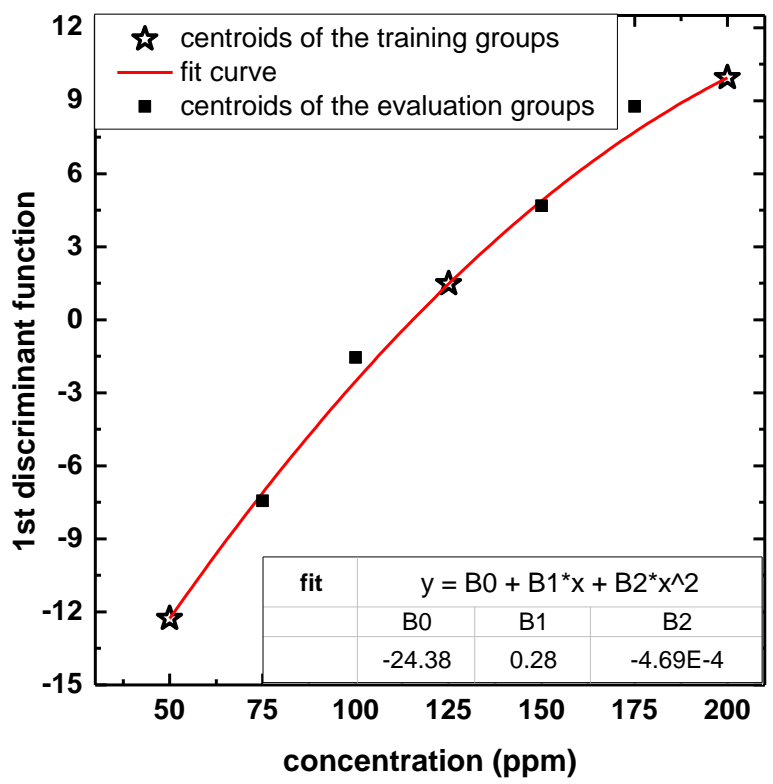

(b)

Fig. 7 Prediction of unknown $\mathrm{NH}_{3}$ concentrations in $20 \%$ oxygen. (a) LDA plot of the discrimination. Training data are marked by solid symbols while open symbols represent a projection of unknown $\mathrm{NH}_{3}$ concentration. (b) Dependency of the value of the first DF and the concentration. Training data are marked by stars and fitted by a second order polynomial. Unknown concentrations (marked by solid squares) fit well to the training.

instance due to temperature effects or other side effects while exposing the sensor to gas. In these cases the actual sensor reaction is not reflected by the features.

Another important point is the use of robust, gas sensitive features. Features reflecting absolute signal values e.g. minimum and maximum are usually not appropriate since drift and outliers will be enhanced. In contrast features describing the shape of the sensor signal e.g. slope, mean value or the Euclidean norm of the data point vector are much more stable and should therefore be preferred and hence, making a classification more reliable.

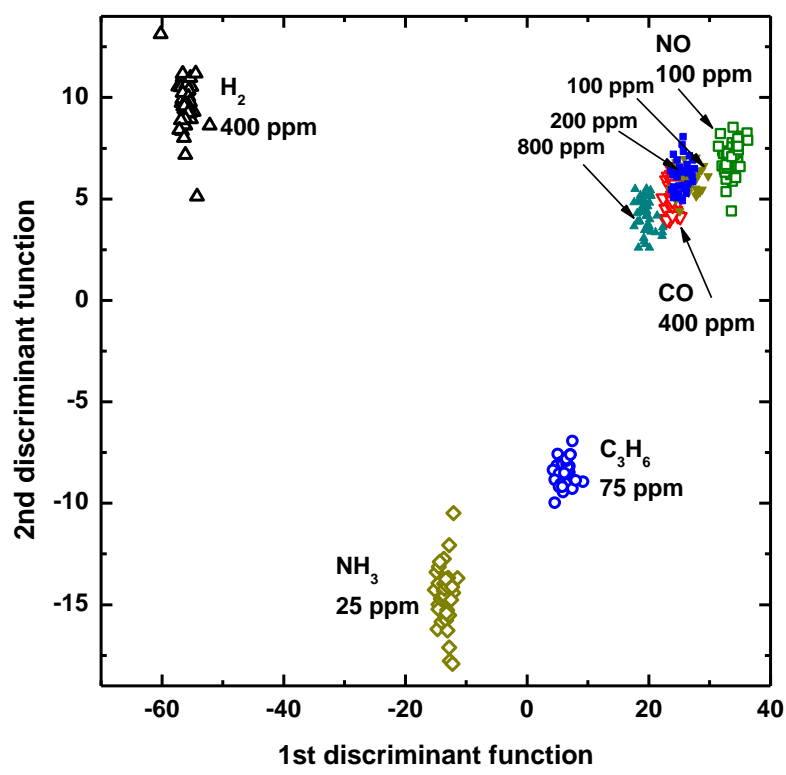

Fig. 8 Verification of the T-cycle approach. LDA coefficients from separation of different gases are applied to various concentrations of $\mathrm{CO}$ in an atmosphere with $20 \%$ oxygen in the background.

In order to validate the features selected for this work, discrimination of different gases is treated again as shown earlier in Fig. 5d. The obtained LDA coefficients from the discrimination of the different gases (where each group contains only one concentration) are applied to different concentrations of e.g. CO (Fig. 8 solid symbols). It can be observed that the new groups representing the different concentrations of $\mathrm{CO}(100,200$ and $80 \mathrm{ppm})$ are fairly close to the 400 ppm CO group used for calculating the LDA. Thus, the selected features actually represent the characteristics of the gas and not e.g. the absolute sensor signal or other side effects. With this, the use of temperature cycling as a suitable operation mode for MISiC FET is proven.

\section{F. Stability and Reproducibility}

When working with chemical sensors there is always the question about stability and reproducibility because these are main drawbacks of many sensors.

In this context the expression "stability" means how the sensor response changes over time. This has been investigated by a long measurement with $\mathrm{NH}_{3}$ and $\mathrm{H}_{2}$ exposures at three points in time with 8 hour breaks in between. The measurement protocol is shown in the upper part of Fig 9. The carrier gas is oxygen in nitrogen and the latter is also defined as an additional group, also at three points in time. Results from an LDA trained to discriminate all nine classes are shown in the lower part of Fig. 9. Obviously, the different gases are separated by the first discriminant function while the second DF discriminates the different points in time, i.e. reflecting the sensor drift. The groups corresponding to the first gas exposure are separated from the second and third while the groups of the last two exposures show considerable overlap. This can be explained by the fact that the sensor needs some kind of "run-in-time". After that the sensor response is fairly 

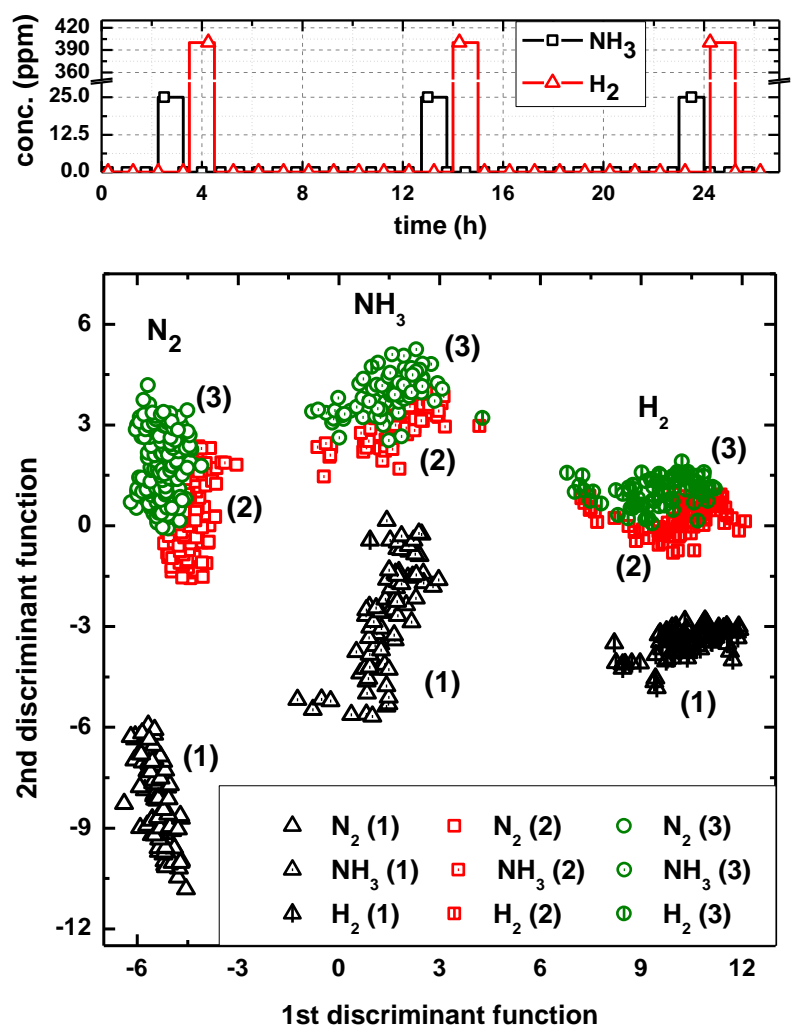

Fig. 9 Investigation on the stability of MISiC FET operated by dynamic temperature modulation. The used gases are $25 \mathrm{ppm} \mathrm{NH}_{3}$ with $10 \%$ oxygen and $400 \mathrm{ppm} \mathrm{H}_{2}$ with $20 \%$ oxygen in the background exposed at three different points in time.

stable.

Reproducibility was checked by repeating measurements with different $\mathrm{H}_{2}$ concentrations after one week under nearly identical conditions. The original measurement was evaluated by LDA (Fig. 10, open symbols) and the resulting LDA coefficients were applied to the data of the repeat measurement (Fig. 10, half-solid symbols).

There is a small shift visible between the original groups and the new ones obviously caused by sensor drift. In this case, the shift is homogenous, i.e. all groups are shifted to the right along DF 1 and a recalibration as reported in [31] should be suitable to eliminate this drift.

\section{CONCLUSION}

The purpose of this paper was to study the potential of dynamic temperature modulation applied to MISiC FET gas sensors. Measurements with typical exhaust gases under laboratory conditions using this approach, originally applied to semiconductor gas sensors, have also shown promising results with a Pt-gate MISiC FET, so that an improvement of selectivity and stability can be achieved.

In a first step a suitable temperature cycle for the detection of exhaust gases $\left(\mathrm{C}_{3} \mathrm{H}_{6}, \mathrm{NO}, \mathrm{CO}\right.$ and $\left.\mathrm{H}_{2}\right)$, as well as ammonia, was developed and significant features to describe the sensor signal were defined. Linear discriminant analysis (LDA) as a pattern recognition tool turned out to be suitable for evaluation of the measurement data. Both, qualitative analysis for

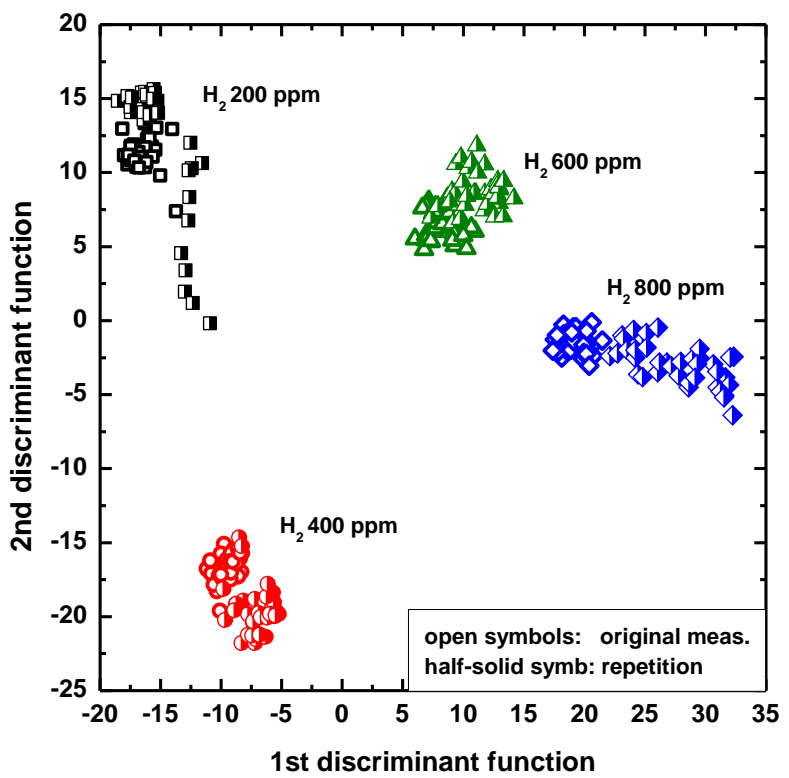

Fig. 10 Investigation on the reproducibility of different concentrations of $\mathrm{H}_{2}$ with $20 \%$ oxygen in the background. Open symbols: original measurement, half-solid symbols: repetition after 1 week.

discrimination of individual gases as well as quantitative determination of gas concentrations with prediction of unknown concentrations have been demonstrated. Background oxygen influences the sensor signal considerably but the characteristic pattern can still be used for evaluation. Water vapor contained in the background changes the sensor behavior a lot compared to the reaction under dry conditions. However, humidity levels higher than $20 \%$ the amount of water has only a small impact on the sensor response pattern and thus the gas discrimination.

Furthermore, we have shown that different gases can be identified characteristically using the extracted features and small changes of the gas concentration do not prevent the identification of the gases.

\section{REFERENCES}

[1] H. Wingbrant, I. Lundström, and A. Lloyd Spetz, "The speed of response of MISiCFET devices," Sens. Actuators B, vol. 93, pp. 286294, 2003.

[2] A. Arbab, A. Lloyd Spetz, and I. Lundström, "Gas sensors for high temperature operation based on metal oxide silicon carbide (MOSiC) devices," Sens. Actuators B, vol. 15, no. 1-3, pp. 19-23, 1993.

[3] G.W. Hunter, P.G. Nuedeck, G.D. Jefferson, C.G. Madzsar, C.C. Liu, and H.Q. Wu, "The development of hydrogen sensor technology," NASA Techn. Memor., vol. 106 141, 1993.

[4] I. Lundström, H. Sundgren, F. Winquist, M. Eriksson, C. KrantzRülcker, A. Llyod Spetz, "Twenty-five years of field effect gas sensor research in Linköping," Sens. Actuators B, vol. 121, pp. 247-262, 2007.

[5] M. Andersson, P. Ljung, M. Mattsson, M. Löfdahl, and A. Lloyd Spetz, "Investigations on the possibilities of a MISiCFET sensor system for OBD and combustion control utilizing different catalytic gate materials," Top. Catal., vol. 30/31, pp. 365-368, 2004.

[6] M. Koebel, M. Elsener, and M. Kleemann, "Urea-SCR: A promising technique to reduce $\mathrm{NO}_{\mathrm{x}}$ emissions from automotive diesel engines," Catal. Today, vol. 59, pp. 335-345, 2000.

[7] H. Wingbrant, H. Svenningstorp, P. Salomonsson, D. Kubiniski, J. H. Visser, M. Löfdahl, and A. Lloyd Spetz, "Using a MISiC-FET Sensor 
for Detecting $\mathrm{NH}_{3}$ in SCR Systems," IEEE Sensors Journal, vol. 5, no. 5, pp. 1099-1105, 2005.

[8] C. Bur, P. Reimann, M. Andersson, A. Lloyd Spetz, and A. Schütze, "New method for selectivity enhancement of SiC field effect gas sensors for quantification of $\mathrm{NO}_{\mathrm{x}}$," Microsystem Technologies, Springer-Verlag, Berlin, submitted for publication

[9] C. Bur, M. Andersson, A. Lloyd Spetz, and A. Schütze, "Hierarchical strategy for quantification of $\mathrm{NO}_{\mathrm{x}}$ in a varying background of typical exhaust gases," Proc. IEEE Sensors 2011, pp. 137-140, Limerick, Ireland, 28-31 Oct., 2011.

[10] O. Larsson, A. Göras, J. Nytomt, C. Carlsson, A. Lloyd Spetz, T. Artursson, M. Holmberg, I. Lundström, L.-G. Ekedahl, and P. Tobias, "Estimation of air fuel ratio of individual cylinders in SI engines by means of MISiC sensor signals in a linear regression model," SAE 2002, 2002-01-0847, Detroit, USA, 4-7 March 2002, also selected for SAE 2002 Transactions - Journal of Engines.

[11] M. Andersson, L. Everbrand, A. Lloyd Spetz, T. Nyström, M. Nilsson, C. Gauffin, and H. Svensson, "A MISiCFET based gas sensor system for combustion control in small-scale wood fired boilers," Proc. IEEE Sensors 2007, pp. 962-965, Atlanta, USA, 28-31 Oct. 2007.

[12] SenSiC AB, Isafjordsgatan 39B, SE-164 40 Kista, Sweden, www.sensic.se

[13] Th. Classen, and K. Sahner, "Trends in automotive exhaust gas sensing," Proc. Sensor+Test Conf. 2011, pp. 554-557, Nurmberg, Germany, 7-9 June 2011.

[14] A. Gramm, and A. Schütze, "High performance solvent vapor identification with a two sensor array using temperature cycling and pattern classification,” Sens. Actuators B, vol. 95, pp. 58-65, 2003.

[15] H. Eicker, "Method and apparatus for determining the concentration of one gaseous component in a mixture of gases," US Patent, no. 4012692, 1977.

[16] A.P. Lee, and B.J. Reedy, "Temperature modulation in semiconductor gas sensing," Sens. Actuators B, vol. 60, pp. 35-42, 1999.

[17] W. M. Sears, K. Colbow, and F. Consadori, "General characteristics of thermally cycled tin oxide gas sensors," Semicond. Sci. Technol., vol. 4, pp. 351-359, 1989.

[18] W. M. Sears, K. Colbow, and F. Consadori, "Algorithms to improve the selectivity of thermally cycled tin oxide gas sensors," Sens. Actuators B, vol. 19, pp. 333-349, 1989.

[19] R.O. Duda, P.E. Hart, D. and G. Stork, "Pattern classification," 2nd ed., Wiley, New York, 2000.

[20] A. Schütze, A. Gramm, and T. Rühl, "Identification of organic solvents by a virtual multisensor system with hierarcical classification," Proc. IEEE Sensors 2002, pp. 382-387, Orlando, USA, 12-14 June 2002.

[21] J. Schalwig, P. Kreisl, S. Ahlers, and G. Müller, "Response mechanism of SiC-Based MOS field-effect gas sensors, "IEEE Sensors Journal, vol. 2, no. 5, pp. 394-402, 2002.

[22] M. Andersson, H. Wingbrant, and A. Lloyd Spetz, "Study of the CO Response of SiC based Field Effect Gas Sensors," Proc. IEEE Sensors 2005, pp.105-108, Irvine, CA, USA, 30 Oct.-3 Nov. 2005.

[23] M. Andersson, and A. Lloyd Spetz, "Detecting non-hydrogen containing species with field effect devices," Proc. IEEE Sensors 2008, pp.13201323, Lecce, Italy, 26-29 Oct. 2008.

[24] ACREO AB, Kista, Sweden, www.acreo.se

[25] Heraeus Holding GmbH, Hanau, Germany, www.heraeus-sensortechnology.com

[26] R. Gutierrez-Osuna, "Pattern analysis for machine olfaction," IEEE Sensors Journal, vol. 2, no. 3, pp. 189-202, 2002.

[27] A. Savitzky, and M.J.E. Golay, "Smoothing and differentiation of data by simplified least squares procedures," Anal. Chemistry, vol. 36, no. 8, pp. 1627-1639, 1964.

[28] C. Bur, P. Reimann, M. Andersson, A. Lloyd Spetz, and A. Schütze, "Temperature cycled operation of $\mathrm{SiC}$ field effect gas sensors: increasing the selectivity for improved sensor systems, " ISOEN 2011, New York, USA, 02-05 May, AIP. Conf. Proc. vol. 1362, pp. 91-92, 2011.

[29] A. Fort, M. Mugnaini, I. Pasquini, S. Rocchi, and V. Vignoli, "Modeling of the influence of $\mathrm{H}_{2} \mathrm{O}$ on metal oxide sensor responses to CO,” Sens. Actuators B, vol. 159, pp. 82-91, 2011.
[30] R.G. Pavelko, A.A. Vasiliev, E. Llobet, X. Vilanova, V.G. Sevastyanov, and N.T. Kuznetsov, "Selectivity problem of metal oxide based sensors in the presence of water vapor," Proc. Eurosensors XXIV, pp.111-114, Linz, Austria, 05-08 Sep., 2010.

[31] P. Reimann, S. Horras, and A. Schütze, "Field-test system for underground fire detection based on semiconductor gas sensor," Proc. IEEE Sensors 2009, pp. 659-664, Christchurch, New Zealand, 25-29 Oct. 2009.

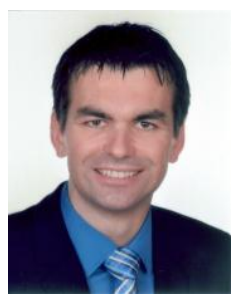

Christian Bur (M'11) received his diploma in Mechatronics, specifically Microtechnology and sensor science, from Saarland University, Saarbrücken, Germany, in 2011.

$\mathrm{He}$ is currently enrolled in the European doctoral program DocMASE presuming a double Ph.D. between Saarland University and Linköping University, Linköping, Sweden. His main research interests are in the field of field effect based gas sensors for emission control with temperature modulation and appropriate signal processing.

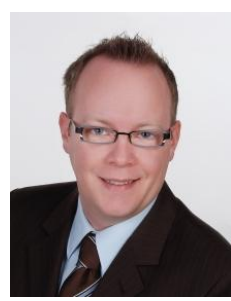

Peter Reimann received his diploma in Electrical Engineering and his Ph.D. from Saarland University, Saarbrücken, Germany in 2006 and 2011, respectively.

His research focus was gas measurement systems for safety and security applications especially for fire detection combined with self-monitoring strategies. After completing his Ph.D. he joint Dillinger Hütte GTS, D-66763 Dillingen/Saar, Germany, where he is working for central services.

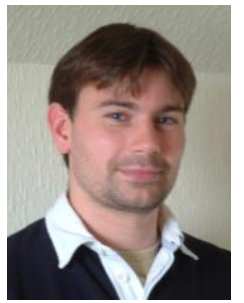

Mike Andersson received the M.Sc. degree in engineering and the Ph.D. degree in applied physics, specifically sensor science, from Linköping University, Linköping, Sweden, in 2002 and 2007, respectively.

Following two Postdoctoral years, he is currently employed as a Research Scientist at Linköping University. His main research interests are in the field of semiconductor based gas sensors for hightemperature applications.

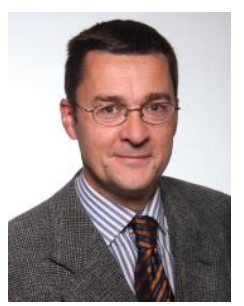

Andreas Schütze (M’01) received his diploma in physics from RWTH Aachen in 1990 and his doctorate in Applied Physics from Justus-Liebig-Universität in Gießen in 1994 with a thesis on microsensors and sensor systems for the detection of reducing and oxidizing gases.

From 1994 until 1998 he worked for VDI/VDE-IT, Teltow, Germany, mainly in the fields of microsystems technology. From 1998 until 2000 he was professor for Sensors and Microsystem Technology at the University of Applied Sciences in Krefeld, Germany. Since April 2000 he is professor for Measurement Technology in the Department of Mechatronics at Saarland University, Saarbrücken, Germany and head of the Laboratory for Measurement Technology (LMT). His research interests include microsensors and microsystems, especially intelligent gas sensor systems for security applications.

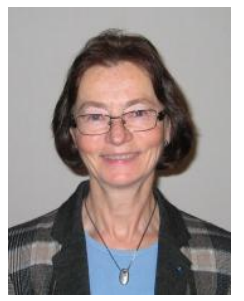

Anita Lloyd Spetz is a Professor of Sensor Science, Chemical Sensors at Linköping University, Linköping, Sweden (2011-2014), and part-time (50\%) FiDiPro Professor at Oulu University, Finland. Since 2007, she is the vice director of VINN Excellence Centre, FunMat, Functional Nanostructured Materials. From 1995 to 2006, she was at S-SENCE, Swedish Sensor Centre (Centre of Excellence). She runs application projects with industry, and she is a Member of the Board of SenSiC AB for commercialization of SiC-FET sensors. Her research involves SiC-FET high-temperature gas sensors with catalytic sensing layers and MAX material ohmic contacts, wide band-gap material transducers for bio-, resonator-, soot-, and graphene/SiC sensors.

(http://people.ifm.liu.se/spetz/). 